Une publication dans une revue scientifique sert à diffuser un travail auprès de la communauté internationale. La revue est une vitrine, cruciale pour le chercheur ainsi que pour son employeur, souvent une institution publique. Une publication se doit donc de satisfaire à de nombreux critères de qualité : une validation rigoureuse par les pairs, un accès facile et pérenne, une indexation dans les bases de données..., toutes choses qui requièrent un travail professionnel.

Cependant, ces dernières années, le coût et le nombre de publications ont explosé et de nombreuses revues en accès libre ont émergé. Ces revues, financées en amont par les auteurs ou leurs institutions, sont-elles plus adaptées aux besoins des chercheurs? Les institutions sont-elles prêtes à les financer ? dans le glossaire, p. 31 .

\title{
Réflexions sur la « Voie dorée » des publications scientifiques
}

Agnès Henri ${ }^{\text {(a) }}$ (agnes.henri@edpsciences.org), Bart van Tiggelen $^{(b)}$ et Marie-Anne Leriche ${ }^{(t)}$ (a) Directrice éditoriale, EDP Sciences.

(b) Président de la Commission des Publications de la Société Française de Physique.

(c) Cellule nationale de l'Information Scientifique et Technique du CEA.

\section{Publish or perish}

La publication d'un article scientifique est une étape cruciale de la recherche. Elle rend visible le résultat d'un travail scientifique, qui sera évalué, commenté, puis cité par les pairs. Les chercheurs, les unités de recherche, et même les organismes et les universités, sont évalués sur la qualité et l'impact de leurs publications. C'est pour cette raison que l'accessibilité, la visibilité et la pérennité d'une publication sont essentielles. Plus encore, puisque la recherche a été souvent soutenue par des crédits publics, les publications qui en résultent se devraient même d'être ouvertement accessibles ; c'est en tout cas un engagement international, signé par la plupart des organismes de recherche $\mathrm{e}^{(1)}$ et rendu possible par l'avènement d'Internet.

Cependant, mettre en œuvre des publications de qualité, visibles et pérennes, même sans version imprimée, requiert un travail d'édition professionnel ${ }^{(2)}$ avec un coût qui peut être chiffré( ${ }^{(3)}$. Comment alors prendre en compte ces besoins dans le système traditionnel d'abonnement où il $\mathrm{y}$ a peu d'accès libre et, plus encore, où l'accès aux revues est quelquefois impossible du fait de coûts d'abonnements trop élevés pratiqués par certains éditeurs ? Le manifeste de l'Université de Californie contre les prix des abonnements du Nature Publishing Group ${ }^{(4)}$ ou, plus récemment, le rejet par l'Université de Harvard des abonnements aux revues de Reed-Elsevier ${ }^{(5)}$ sont deux signes qui montrent que le modèle classique des revues sous abonnement atteint ses limites.

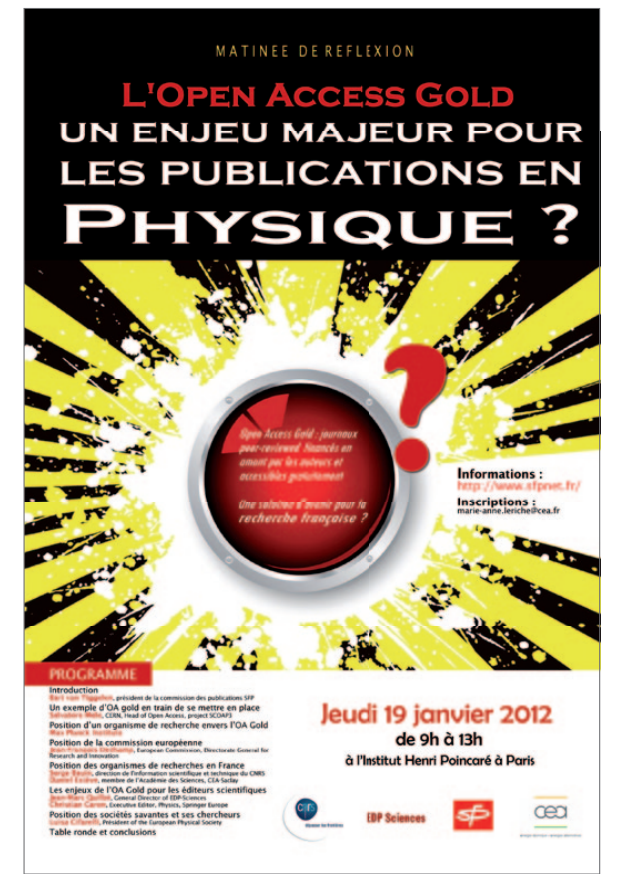

Affiche de la matinée OAG du 19 janvier 2012.

\section{Open Access Green et Open Access Gold}

Les archives ouvertes (HAL ${ }^{\star}$ en France), dites la "Voie verte ", sont une première étape vers le libre accès. Elles sont surtout appréciées par les chercheurs en amont d'une publication, dans le processus de prépublication, et autorisées par la plupart des éditeurs. En revanche, l'article déposé n'est ni soumis à une évaluation scientifique par les pairs ("peer review"), ni indexé dans les bases de données consultées par les comités d'évaluation ${ }^{(6)}$. D'autre part, pour certaines communautés, mettre en accès libre des résultats de recherche non validés pose un vrai problème. C'est le cas du domaine médical, ou encore celui du nucléaire : on comprend bien le danger de mettre à disposition du public des résultats sensibles ou non validés.

Parallèlement à ces archives ouvertes, nous avons tous pu constater ces dernières années une nette accélération des publications en Open Access Gold, OAG* ("Voie dorée ", voir l'encadré p. 31). 


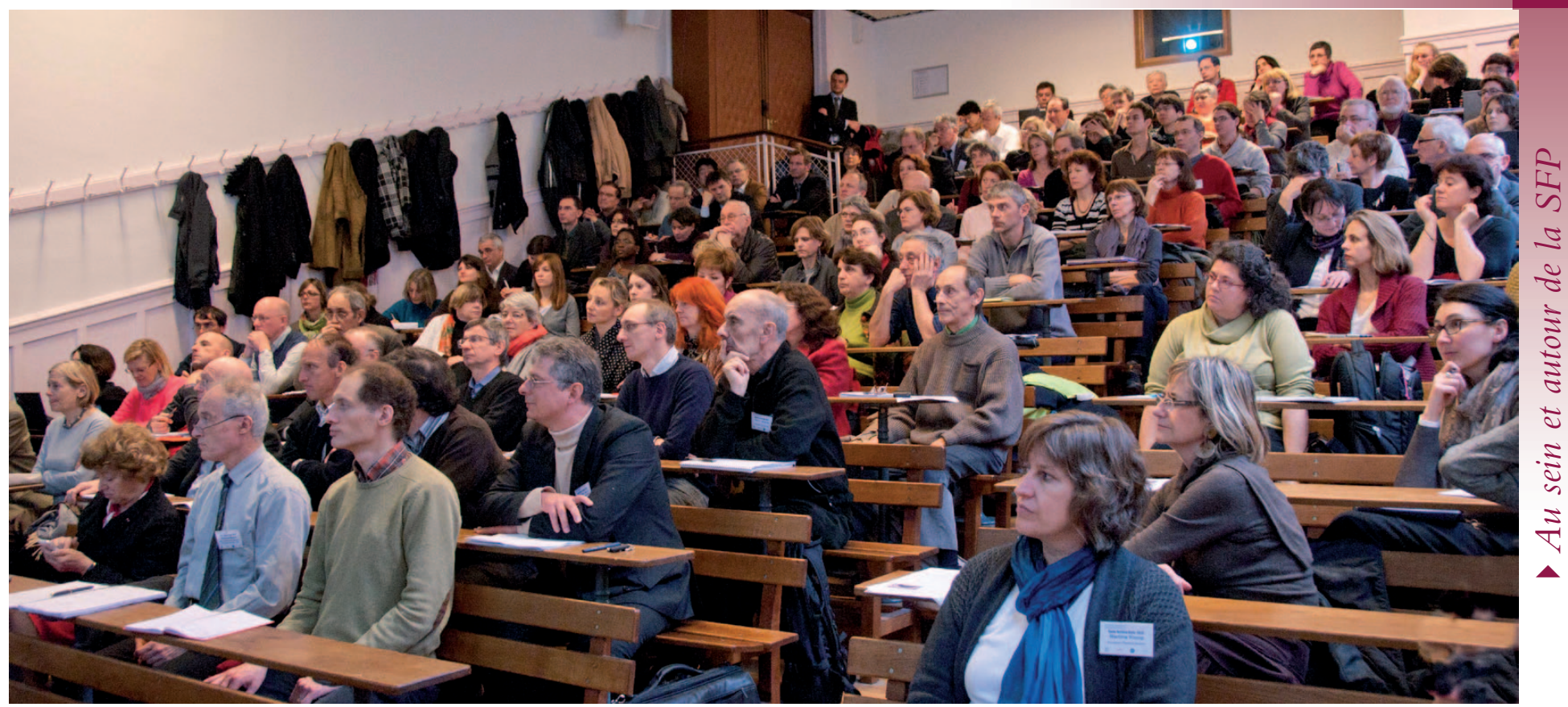

Matinée de réflexion OAG à l'Institut Henri Poincaré, le 19 janvier 2012.

La Société Française de Physique, très impliquée dans les publications avec sa maison d'édition EDP Sciences, coéditeur de la plateforme EPJ et de la revue de lettres EPL, a donc souhaité organiser une matinée de réflexion, en association avec le CEA et le CNRS (l'ensemble des présentations et le compte rendu complet sont disponibles sur www.openaccessgold.org). En effet, la France semble être en retard sur le débat de l'OAG et de son financement, comparé à d'autres pays, plus particulièrement les pays anglo-saxons. L'enjeu de cette matinée, qui s'est tenue à Paris à l'Institut Henri Poincaré le 19 janvier dernier, était de permettre aux participants de partager leurs expériences sur des projets et des développements sur l'Open Access menés au sein d'autres pays, et de lancer en France la discussion sur la Voie dorée, en incitant les organismes de recherche français à prendre position. Cette matinée fut un succès, et la communauté - chercheurs, bibliothécaires, documentalistes, éditeurs, dirigeants des organismes - a répondu présente, avec plus de 100 participants !

\section{La Voie dorée : où en sommes-nous aujourd'hui ?}

Le consortium SCOAP3*, porté par le CERN, est une opération potentiellement exemplaire en matière d'OAG. Elle a été mise en ouvre par les chercheurs de la physique des hautes énergies dans le but précis de mettre en libre accès les publications du CERN, avec l'implication des bibliothèques et des maisons d'éditions de toutes nationalités (en France, on note l'implication de l'IN2P3). Lancé il y a maintenant 5 ans, SCOAP3 a aujourd'hui bouclé son financement (10 M€/an) à 80\%. Le consortium a ainsi négocié directement avec les revues de son domaine le libre accès de leurs publications, les revues restant les garantes de la qualité du peer review.

Quelle vision de l'Open Access Gold ont des organismes comme le CNRS et le CEA ? La Direction de l'Information Scientifique et Technique (DIST) du CNRS, par la voix de son directeur Serge Bauin, a expliqué qu'il n'y a pas de politique officielle du CNRS sur la Voie dorée. D'abord parce que la Voie verte semble toujours très soutenue et partagée au sein du CNRS, stimulée encore davantage par le comportement agressif et commercial de certaines grandes maisons d'édition évoqué auparavant. Ensuite, la nature très pluridisciplinaire du CNRS rend difficile l'émergence d'une position consensuelle sur la Voie dorée, bien que certains instituts (l'IN2P3 et certains domaines de la biomédecine) soient déjà très avancés sur le sujet. Au CEA, le débat semble plus avancé et plus chiffré. L'OAG représente environ 5\% de ses publications, en sachant que ce pourcentage est très variable en fonction des disciplines et peut atteindre $20 \%$ pour les sciences du climat et de l'environnement. Même si dans le domaine de la physique, ArXiv répond en partie au besoin de libre accès, le CEA reste attaché à une publication par ses chercheurs dans des revues de qualité, qu'elles soient en OAG ou non.
Aujourd'hui, la majorité des éditeurs scientifiques se sont lancés sur la Voie dorée. Alors qu'en 2007, le DOAJ^ répertoriait 3000 journaux de ce type, aujourd'hui, plus de 7700 revues en libre accès y sont recensées (sur un total de 25400 en 2009, donc presque un tiers des revues sont en OAG). On estime une croissance d'OAG entre $20 \%$ et $30 \%$ par an ${ }^{(7)}$. Différents événements, comme la Déclaration de Berlin $^{(8)}$ en 2003, la nette préférence pour l'OAG affirmée par la Max Planck Gesellschaft (accompagnée d'un soutien financier pour ses auteurs souhaitant publier dans des revues OAG, en opposition au modèle hybride) et le CERN avec SCOAP3, ont amené les éditeurs scientifiques à se lancer sur la voie hybride, puis sur la Voie dorée. La voie hybride ne peut être que transitoire, car dans ce modèle économique les institutions ont l'impression de payer deux fois : une fois pour les abonnements, et une fois pour l'accès libre.

Selon le PDG d'EDP Sciences, Jean-Marc Quilbé, la mise en œuvre de la Voie dorée est difficile et surtout risquée pour un éditeur de taille moyenne comme EDP Sciences, et ce, principalement tant que la plupart des communautés scientifiques et des organismes de recherche ne se sont pas exprimés sur leur manière de le financer. Pour une grande maison d'édition comme Springer, il a été plus facile d'amorcer un virage vers la Voie dorée, Springer s'étant d'ores et déjà positionné comme un acteur majeur de l'OAG grâce au rachat de BioMed Central (éditeur de plus de 230 revues en OAG dans le domaine de la médecine et 


\section{\>}

de la biologie, une communauté utilisant peu ou pas ArXiv). Les coûts de publication d'un article y varient de $500 €$ à $2000 €$ selon les revues. Springer vient par ailleurs de lancer la plateforme "dorée "Springer Open et, avec EDP Sciences et la Société Italienne de Physique, plusieurs revues en Open Access sur la plateforme EPJ ${ }^{(9)}$. Pour la communauté des physiciens, l'arrivée en 2011 de grands acteurs comme l'American Physical Society avec Physical Review X ou de Nature avec Scientific Reports a démontré, s'il le fallait encore, l'ampleur de ce mouvement OAG, après le lancement, dès 1999, du New Journal of Physics (NJP) par la DPG (Deutsche Physikalische Gesellschaft) et l'IOPP (Institute of Physics Publishing) ${ }^{(10)}$.

Au Royaume-Uni, le débat public sur l'OAG est très avancé. Le 8 décembre dernier, le ministre britannique de la recherche prenait résolument position pour le libre accès à la recherche $e^{(11)}$, et un "Wellcome Trust"(12) existe pour aider à son financement. Des études récentes au Royaume-Uni ont estimé que le basculement vers l'OAG pourrait se faire à la condition que le coût d'une publication ne dépasse pas la somme de $1450 €^{(13)}$. Pourtant, la directrice éditoriale de l'IOPP, Nicola Gulley, estime, pour sa part, le coût réel d'une publication plus élevé : autour de $2400 €$. La question se pose donc du coût réel d'une publication et de la « juste " rémunération de l'éditeur.

La Commission Européenne insiste sur le juste retour de l'utilisation des fonds publics investis dans la recherche, et sur la cohérence de la politique du libre accès entre les différents pays membres. Elle a donc mis en place des actions en faveur de l'Open Access dans le $7^{\mathrm{e}}$ programme cadre (FP7), mais sans pour autant obliger les chercheurs à suivre la Voie dorée. La Commission souhaite qu'à partir de 2014 , toutes les publications issues de recherches qu'elle a financées, par exemple via l'ERC (European Research Council), soient en Open Access. La Commissaire européenne Neelie Kroes, chargée du dossier numérique, est très favorable à l'accès libre des publications, et admet le fait qu'il va beaucoup plus loin que la simple mise en ligne d'un fichier pdf dans une archive ouverte, en reconnaissant le rôle majeur des maisons d'édition pour la diffusion des travaux de recherche $\mathrm{e}^{(14)}$

\section{Avantages et inconvénients de la Voie dorée}

Pour les adversaires de l'OAG au sein de la communauté scientifique, le modèle OAG a quelques ambiguïtés. La Société Européenne de Physique (EPS), avec sa présidente actuelle Luisa Cifarelli, a présenté sa position sur l'Open Access dans un article publié en $2009^{(15)}$, et y mettait déjà en évidence quelques points délicats au sein du modèle économique de l'OAG. Dans la formule " auteur-payeur ", un doute existe sur l'objectivité du processus d'acceptation d'un éditeur commercial, car les revenus sont directement proportionnels au nombre d'articles acceptés alors que la qualité scientifique, "le prestige " quantifié par le facteur d'impact, y est inversement proportionnel. Évidemment, pour les éditeurs qui sont propriétés des sociétés savantes, comme EDP Sciences, APS, IOPP, ou proches, de même que certaines revues de Springer, la qualité sera toujours sous contrôle grâce à l'implication directe des chercheurs, et donc moins exposée aux intérêts commerciaux.

Un des points forts mis en avant par la Voie dorée est la libéralisation des droits d'auteur. Dans le modèle d'abonnement, l'éditeur se rémunère avec la vente des publications aux lecteurs. Or, le transfert des droits d'auteur, donnant à l'éditeur l'entière propriété du contenu et donc le droit de vente, a toujours été un point délicat pour les chercheurs. Mais dans la formule OAG, le transfert des droits d'auteur n'est plus nécessaire puisque le paiement se fait en amont par l'auteur ou son institution. Ainsi, les droits d'exploitation du contenu restent à l'auteur ; plusieurs éditeurs (Springer, EDP Sciences, IOPP) utilisent donc des licences dites creative commons pour les publications dorées, qui permettent ainsi une réutilisation facile des contenus par les auteurs ou les lecteurs, à la condition que la référence de l'article soit correctement citée. Un auteur pourra, par exemple, réutiliser une partie de sa publication ou la mettre à disposition de tous sur son site, sans demander l'autorisation à l'éditeur.

Une question clé, surtout pour les organismes de recherche, est de savoir si la Voie dorée est en mesure de stopper la flambée des prix des publications. Une première explication de cette flambée est l'attitude commerciale de certains éditeurs, en situation de monopole, qui vendent de grands packages de revues qui ne sont pas toujours souhaités par l'abonné. La Voie dorée a l'avantage de faire disparaître ce type de situation, puisqu'il n'y a plus d'abonnés. Une deuxième explication à cette augmentation des prix est la simple explosion du nombre des publications : une étude de 2009 montrait que le nombre de publications a augmenté de 3,5\% par an ces deux dernières décennies ${ }^{(6)}$; et l'arrivée sur le marché des pays du BRIC* ne fait qu'accélérer le processus. La revue EPL illustre bien ce phénomène, puisque le nombre de soumissions (2400 en 2011) a doublé depuis 2006. Certaines viennent de Chine, pays qui, par ailleurs, est peu enclin à souscrire des abonnements. Dans le modèle traditionnel de publication, ce sont les bibliothèques qui paient cette augmentation via les abonnements.

D'une part, la Voie dorée permet l'émergence de revues publiant des articles sans aucune limite, puisque les frais de publication sont pris en charge par les auteurs (ou leur institution) et non par les bibliothèques (dans le modèle classique, le nombre d'articles publiés par l'éditeur est limité par le chiffre d'affaires des abonnements). C'est le cas de PLOS ONE (revue OAG généraliste dans le domaine de la médecine et de la biologie) et, plus récemment, de SpringerPlus. Parmi ces journaux (publiant souvent des séries de données, avec un peer review réduit), PLoS ONE rencontre un grand succès en termes d'impact et de revenu, malgré une exigence de qualité de publication assez réduite. Ce type de journal semble donc répondre à un certain besoin de la communauté, sans pour autant toucher les budgets des bibliothèques. Mais cela rejoint les craintes de l'EPS évoquées plus haut : un modèle économique où les revenus sont directement proportionnels au nombre d'articles acceptés, poussant à (presque) tout accepter.

D'autre part, pour les revues OAG de " prestige " (c'est-à-dire avec un taux de rejet élevé, en sélectionnant les articles qui sont potentiellement de haut impact), on constate à nouveau une flambée des prix d'un article (3570€ pour Nature Communications). Dans un modèle entièrement commercial, que les revues soient en $\mathrm{OAG}$ ou non, plus elles sont prestigieuses, plus elles sont chères, le prestige étant souvent récompensé par les institutions et les évaluateurs. L'action récente de l'Université de Californie contre 
Nature Physics Group est peut-être un premier signe que ce " cercle vicieux " est en train de se briser.

\section{II est temps de lancer le débat en France}

Le moment est venu de reconsidérer la façon de publier les résultats issus de nos recherches scientifiques. Les recherches, y compris leur diffusion, sont pour la plupart soutenues par des crédits publics, mais pas toujours ouvertement accessibles. Le chercheur exige le plus large impact possible de ses travaux, le plus longtemps possible, car sa carrière en dépend. La qualité des revues doit être garantie par l'implication directe de ses "pairs " ou par les sociétés savantes qui défendent leurs intérêts. Pour la mise en œuvre, le chercheur aura toujours besoin d'éditeurs professionnels, qui demandent un juste prix pour leurs efforts, en fonction du travail effectué. Le modèle traditionnel de l'édition scientifique, avec les abonnements, souvent très chers pour les revues de prestige, souffre clairement de quelques défaillances, et il faut donc le faire évoluer.

Il est très important que les organismes de recherche en France s'engagent sur une nouvelle politique pérenne concernant le mode de publication. Pour avoir des chances de réussir la nécessaire évolution de l'édition scientifique, il semble impératif d'associer à la réflexion tous les partenaires impliqués : chercheurs, institutions, évaluateurs, éditeurs, financeurs de la recherche, non seulement au plan français, mais également au plan européen. L'action concertée d'un tel groupe de partenaires aura la force de faire basculer l'ancien monde de l'édition vers un système durable pour l'avenir.

\section{Quelques rappels sur 1'Open Access}

L'Open Access est la mise à disposition en ligne et en libre accès de contenus numériques. Ces contenus peuvent eux-mêmes être soit libres de droits (c'est-à-dire réutilisables facilement sans demander l'autorisation de l'éditeur, mais en citant la source), soit sous un régime de propriété intellectuelle (droits détenus par l'éditeur). On dénombre trois types d'Open Access :

- I'Open Access Green (également appelé « auto-archivage ») :

l'auteur (ou une autre personne agissant en son nom) dépose une version de l'article (article soumis, article approuvé, versions successives, mais souvent pas la version publiée) dans un répertoire ouvert, un site d'institution ou un site personnel. Exemples d'archives ouvertes: HAL* mis en place par le CNRS, suivi plus tard par le CEA, et ArXiv dont I'Université de Cornell est propriétaire. Les revues sous abonnements coexistent avec les archives ouvertes et la plupart des éditeurs autorisent les dépôts des articles dans leur version preprint, avec ou sans embargo. La politique varie selon les éditeurs et est disponible sur le site Sherpa (www.sherpa.ac.uk/romeo/) ou, en France, sur le site Héloise (http://heloise.ccsd.cnrs.fr/) ;

- I'Open Access Hybrid :

au sein d'une revue sous abonnement, seuls certains articles, du fait du choix de l'auteur, sont en libre accès. L'éditeur propose cette option contre un paiement des frais de publication. L'objectif au départ était de permettre le basculement progressif d'une revue sous abonnement à une revue purement Open Access. Dans les revues hybrides (comme dans les revues sous modèle d'abonnement classique), les abonnés ont le choix entre un abonnement à la version électronique seule ou à un combiné " version papier + version électronique ». Mais, de plus en plus, les abonnés se tournent uniquement vers la version électronique, la version papier servant seulement à l'archivage, et non plus à la consultation

\section{- I'Open Access Gold:}

ce sont des revues en libre accès, le payeur est soit l'auteur physique, soit une personne morale, une institution, un consortium ou un projet. Elles sont, la plupart du temps, sous forme électronique, même si quelques éditeurs proposent à la vente une version papier pour ceux qui souhaitent la payer, souvent en " impression à la demande ».

Une étude récente réalisée sur un échantillon de 1837 articles publiés en 2008, toutes disciplines confondues, montrait que $20,4 \%$ des articles étaient en libre accès, dont $8,5 \%$ depuis des sites d'éditeurs (Open Access Gold ou modèle hybride) et 11,9\% en OA green [B. C. Björk et al., PLoS ONE 5 (2010) e11273].

\section{Glossaire}

BRIC : Brésil, Russie, Inde et Chine.

DOAJ : Directory of Open Access Journals, www.doaj.org/

HAL : Hyper Articles en Ligne, http://hal-archives-ouvertes.fr/ et http://hal-cea.archives-ouvertes.fr/ OAG : Open Access Gold (ou « Voie dorée »).

SCOAP3 : Sponsoring Consortium for Open Access Publishing in Particle Physics, http://scoap3.org/

(1) Voir le site de l'INIST, http://openaccess.inist.fr/spip.php?article38

(2) Utilisation d'outils professionnels pour gérer les flux de manuscrits ou, comme CrossCheck, repérer les plagiats, le travail éditorial (copy editing), l'intégration de liens croisés, la maintenance d'une plateforme de publication pérenne avec des outils pour les lecteurs, etc.

(3) "Costs and business models in scientific research publishing", The Wellcome Trust (2004),

www.wellcome.ac.uk/stellent/groups/corporatesite/@policy_communications/documents/web_document/wtd003184.pdf

(4) http://today.ucla.edu/portal/ut/uc-libraries-faculty-protest-proposed-160117.aspx

(5) www.lemonde.fr/sciences/article/2012/04/25/harvard-rejoint-les-universitaires-pour-un-boycott-des-editeurs_1691125_1650684.html

(6) "Web of Knowledge", base de données de Thomson-Reuters : http://wokinfo.com/

(7) R. Poynder, “Open Access by Numbers” (2011), www.richardpoynder.co.uk/Open_Access_By_Numbers.pdf

(8) http://oa.mpg.de/lang/en-uk/berlin-prozess/berliner-erklarung/

(9) The European Physical Journal (EPJ), www.epj.org

(10) Pour les membres de la SFP, société savante associée au NJP, la publication en libre accès dans cette revue coûte $935 €$.

(11) "Results of publicly funded research will be open access", www.guardian.co.uk/science/2011/dec/08/publicly-funded-research-open-access

(12) "Open access at The Wellcome Trust", www.wellcome.ac.uk/About-us/Policy/Spotlight-issues/Open-access/index.htm

(13) S. Pinfield et Ch. Middleton, "Open Access Central Funds in UK Universities”, Learned Publishing, 25 (2012) $107-116$.

(14) N. Kroes, "The Challenge of Open Access" (2010), www.openaccess.be/media/docs/NeelieKroes@OpenAIRE.pdf

(15) “Open Access - A position paper of the EPS”, www.eps.org/resource/resmgr/policy/eps_pp_oa_2009.pdf 